Our Hospital is a non-referral hospital without NICU facilities. Neonates meeting the criteria for therapeutic hypothermia need to be transported to a NICU. The diagnostic value of aEEG monitoring in a non-NICU setting is unknown. We hypothesised aEEG monitoring in a non-NICU setting adds value to diagnostic and therapeutic decision making in asphyxiated neonates.

Methods A retrospective analysis was performed on all asphyxiated neonates born from January 2011 untill July 2013 in our hospital. Asphyxia was defined as Apgar score $\leq 5$ after 5 min or rescusitation or ventilation from birth for $10 \mathrm{~min}$ or $\mathrm{pH}<7.0$ and base deficit $>16 \mathrm{mmol} / \mathrm{L}$ or lactic acid $>10.0 \mathrm{mmol} / \mathrm{L}$.

Results We evaluated 57 asphyxiated neonates of which 12 neonates were directly intubated and transported to NICU. In 7 out of $45(15,5 \%)$ asphyxiated neonates the performed aEEG had diagnostic consequences. Finally, 4 out of 7 neonates (9\%) were treated for subclinical seizures $(n=3)$ or therapeutic hypother$\operatorname{mia}(\mathrm{n}=1)$.

Conclusions aEEG monitoring in a non-NICU setting adds diagnostic and therapeutic value in asphyxiated neonates, especially in the recognition of subclinical seizures.

\section{PS-111 WITHDRAWN}

\section{PS-112 ISCHAEMIC NEONATAL STROKE CLASSIFICATION WITH A 3D MAP OF THE ARTERIAL TERRITORIES OF THE INFANT BRAIN}

${ }^{1} \mathrm{C}$ Stephan-Otto, ${ }^{2} \mathrm{G}$ Arca-Diaz, ${ }^{2} \mathrm{~T}$ Agut, ${ }^{2} \mathrm{~A}$ Garcia-Alix. ${ }^{1}$ Unitat de Recerca, Parc Sanitari Sant Joan de Deu FSJD - UB - CIBERSAM, Barcelona, Spain; ${ }^{2}$ Neonatology, Agrupacion Sanitaria Sant Joan de Deu - Hospital Clinic - UB, Barcelona, Spain

\subsection{6/archdischild-2014-307384.406}

Background and aim Prognosis of neonatal Arterial Ischaemic Stroke (AIS) seems to depend strongly on early MRI findings, while standard neurological examination provides limited prognostic value. The extent of a lesion as well as the brain areas involved appear to be the main outcome indicators. Moreover, classification according to arterial irrigation regions has proven useful. We aim to provide a tool for systematic vascular classification by means of a segmented 3D map with standard neonatal dimensions.

Methods A T1 structural MR image as well as a TOF angiography were deformed into a standardised neonatal brain. Brain structures and main arteries were then identified in this brain, and the corresponding arterial territories delineated with the help of published arterial region maps for adults. The standardised brain was then manually segmented, under supervision of three experts, and assembled into a 3-dimensional map.

Results Reliability of the resulting 3D map was assessed by automatically classifying a series of previously diagnosed AIS. Arterial region attribution mistakes were detected in radiology reports in some cases, supporting the need of this kind of tool. A test sample of 25 AIS with heterogeneous distribution and sizes was used to validate the original segmentation. Only minor corrections to territory boundaries were necessary.

Conclusions We propose a systematic method to characterise AIS location and extent in neonates by means of a standard template of arterial territories. Its effectiveness in the assessment of stroke outcome is left as a future research endeavour with an independent, larger sample.

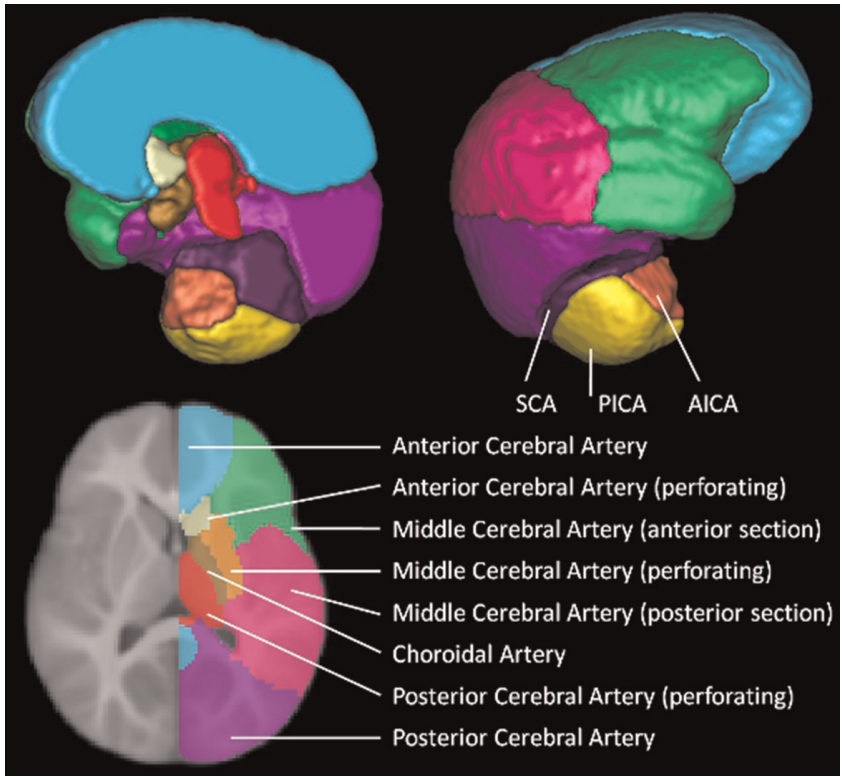

Abstract PS-112 Figure 1

\section{PS-113 THE ASSOCIATION BETWEEN ELECTRICAL BRAIN ACTIVITY AND ARTERIAL SPIN LABELLING PERFUSION MRI IN NEONATES WITH HYPOXIC-ISCHAEMIC ENCEPHALOPATHY (HIE)}

${ }^{1} \underline{S}$ Negro, ${ }^{2} \mathrm{JB}$ de Vis, ${ }^{2} \mathrm{~J}$ Hendrikse, ${ }^{3} \mathrm{~F}$ Groenendaal, ${ }^{3} \mathrm{LS}$ de Vries, ${ }^{2} \mathrm{ET}$ Petersen, ${ }^{3} \mathrm{MC}$ Toet, ${ }^{3} \mathrm{~F}$ van Bel, ${ }^{3} \mathrm{MJNL}$ Benders. ${ }^{1}$ Department of Molecular and Developmental Medicine, University General Hospital of Siena, Siena, Italy; ${ }^{2}$ Department of Radiology, University Medical Centre Utrecht, Utrecht, Netherlands; ${ }^{3}$ Department of Neonatology, University Medical Centre Utrecht, Utrecht, Netherlands

\subsection{6/archdischild-2014-307384.407}

Background and aims (a)EEG predicts outcome in full-term infants with HIE. Recently, increased perfusion in the basal ganglia, detected with arterial spin labelling (ASL), was shown to be related to brain injury ${ }^{1}$. Our aim was to investigate the relationship between (a)EEG and brain tissue perfusion.

Methods 20 subjects with HIE, eligible for hypothermia, were enrolled. Four 1-hour periods were selected from the (a)EEG: P1 (4-6 h), P2 (20-24 h), P3 (32-36 h) and P4 (44-48 h). Burst-rate (number of burst/min) and IBI (interburst interval) from the rawEEG, minimum (MIN) amplitude $(\mu \mathrm{V})$ and the $\%$ of time $<5 \mu \mathrm{V}(\%<5 \mu \mathrm{V})$ of the aEEG, were included in the analysis. Mean perfusion in the basal ganglia and thalami (BGT-CBF) was measured using ASL-MRI.

Results In P1 a relation was found for suppressed aEEG signal $(\%<5 \mu \mathrm{V}: \mathrm{p}=0.015 ; \mathrm{R}=0.709 ; \mathrm{MIN}: \mathrm{p}=0.028 ; \mathrm{R}=$ $0.659)$ and increased BGT-CBF. Concomitantly, a negative correlation was found between burst-rate and BGT-CBF, MIN in P23-4 ( $\mathrm{p}<0.05)$, whereas suppressed background pattern expressed by $\%<5 \mu \mathrm{V}$ and IBI correlated positively with higher BGT-CBF $(\mathrm{p}<0.05)$. In the multivariable regression, corrected for sedatives and anti-epileptic medication, the association between EEG parameters and BGT-CBF persisted $(p<0.05)$, with the exception of burst-rate in P1.

Conclusion A depressed cortical activity in the first $48 \mathrm{~h}$ after birth, secondary to hypoxic-ischemia, is related to an abnormally increased brain perfusion. Using both techniques together might be of additional value to predict neurodevelopmental outcome.

\section{REFERENCES}

1 Wintermark P et al. Am J Neuroradiol 2011;32:2023-29 